\title{
FOPA Pump Phase Modulation and Polarization Impact on Generation of Idler Components
}

\author{
Sergejs Olonkins ${ }^{1}$, Vjaceslavs Bobrovs ${ }^{1}$, Girts Ivanovs ${ }^{1}$ \\ ${ }^{1}$ Institute of Telecommunications, Riga Technical University, \\ Azenes St. 12, LV-1048 Riga, Latvia \\ sergejs.olonkins@rtu.com
}

\begin{abstract}
Fiber optical parametric amplifiers (FOPA) are well known for the variety of potential applications in alloptical signal processing. Many of these applications use the idler spectral components that are produced by the four wave mixing parametric process. The performance of parametric amplifiers and, therefore, also of generation of idler spectral components, is highly dependent on different factors, including stimulated Brillouin scattering (SBS) and polarization of the input signal. The aim of this article is to investigate the influence of pump phase modulation of parametric amplifiers, used for SBS mitigation, and the impact of mutual state of polarization mismatch of the input signal and the pump on the generation of idler spectral components. The obtained results have shown that it is crucial for systems with FOPAs to ensure that the input signal and the pump are co-polarised, as polarization dependant gain of $16.7 \mathrm{~dB}$ and $33.5 \mathrm{~dB}$ difference in idler generation was observed in the performed simulations. It was also found that applying the phase-modulation of the pump for SBS mitigation has caused $54 \%$ idler spectral broadening. Therefore additional measures must be taken to avoid inter-channel crosstalk among the neighbour channels.
\end{abstract}

Index Terms-Fiber optical parametric amplifiers; four wave mixing; idler spectral components; polarization dependant gain; stimulated Brillouin scattering.

\section{INTRODUCTION}

To satisfy the constantly increasing demand for higher network capacity, fiber optical transmission systems with wavelength division multiplexing (WDM) have been intensively studied and applied, as WDM technology allows using the available optical fiber resources more efficiently than alternative technologies [1]-[3]. It is possible to increase the WDM system throughput capacity either by increasing the data transmission speed in channels or the number of channels. Both ways require to enlarge the wavelength band that is used for transmission, but in WDM systems it is limited due to the wavelength dependence of signal attenuation in optical fibers and due to limited amplification wavelength band of conventional erbiumdoped fiber amplifiers (EDFA), that are the dominative type of optical amplifiers used in modern transmission systems [4].

Due to the facts mentioned above, recently a lot of attention has been focused on implementation of alternative

Manuscript received 18 November, 2015; accepted 30 May, 2016.

This research was has been supported by the national research program in Latvia within the project Nr. 10-4/VPP-4/11. types of optical amplifiers in optical transmission systems, especially to fiber optical parametric amplifiers (FOPA). This type of amplifiers, in comparison with conventional EDFAs, can ensure high level of amplification and low noise figure values over relatively large wavelength band, and can ensure amplification almost at any wavelength. Both bandwidth up to $208 \mathrm{~nm}$ [5] and gain up to $70 \mathrm{~dB}$ [6] have been demonstrated.

Due to the variety of potential applications parametric amplification is positioned as the future leading technique for ultrafast all-optical signal processing in optical communication systems [7]. FOPAs can be used for $2 \mathrm{R}$ and 3R signal regeneration [8], wavelength conversion [9], dispersion compensation [10], and so on.

Unfortunately, realization of parametric amplifiers requires implementation of complex solutions, as well as detailed and specific configuration for every particular transmission system. There are several factors that highly limit the performance of FOPAs:

- Inter-channel crosstalk that arises from pump channel four wave mixing interactions (PC-FWM) and from channel-channel interactions (CC-FWM);

- Relative intensity noise (RIN) that is being transferred from the pumps to the amplified signal;

- Scattering of pumping radiation in the backword direction that is caused by initiation of stimulated Brillouin scattering (SBS) nonlinearity in the gain medium;

- High gain dependency on the state of polarization (SOP) of the signal to be amplified;

- Stimulated Raman scattering (SRS) induced energy transfer between the pumps that decreases FWM efficiency.

Previously the authors already have demonstrated how choosing the appropriate pump configuration and implementation of pump phase modulation can help to reduce FWM produced inter-channel crosstalk and to mitigate the impact of SBS on the performance of the FOPA [11]. But in that research the gain polarization dependence was neglected and the impact of such pump-phase modulation on the idler spectral components was not considered. The main advantage of FOPAs over other types of amplifiers is the possibility of using them for all-optical signal processing. Most of such applications of FOPAs are realised through generating idler spectral components. 
Therefore, the aim of this article was to investigate the impact of FOPA gain polarization dependence and of applying pump phase modulation for SBS mitigation, in systems where FOPAs are used for wavelength conversion, on the idler spectral components.

\section{SimULATION SETUPS AND RESULTS}

As it was mentioned previously, there are 2 main goals of this research - investigation of impact of FOPA pump phase modulation and of polarization dependence of the FOPA provided gain on the generated idler spectral components. In both cases numerical simulations were used to obtain experimental results, and in both cases Optilux OptSim 5.3 simulation software was used, as this strong mathematical tool can handle complex simulations of optical transmission systems and it can provide high accuracy results without the requirement for high performance hardware [12].

As mismatch between the states of polarization of the input signal and the pumping radiation can directly impact FWM and, therefore, also the idler generation process, it was decided to analyse gain polarization dependence of parametric amplifiers before estimating the impact of FOPA pump-phase modulation on the idler spectral components.

\section{A. Demonstration of Gain-Polarization Dependence of Parametric Amplifiers}

While obtaining the dependence of idler generation efficiency on the mutual mismatch of the states of polarization between the signal and the pumping radiation, it was decided to simplify the simulation model as much as possible to avoid impact of other factors on the obtained results. For this purpose a simulation model of a singlechannel transmission system with a FOPA preamplifier was introduced. This simulation model is displayed in Fig. 1.
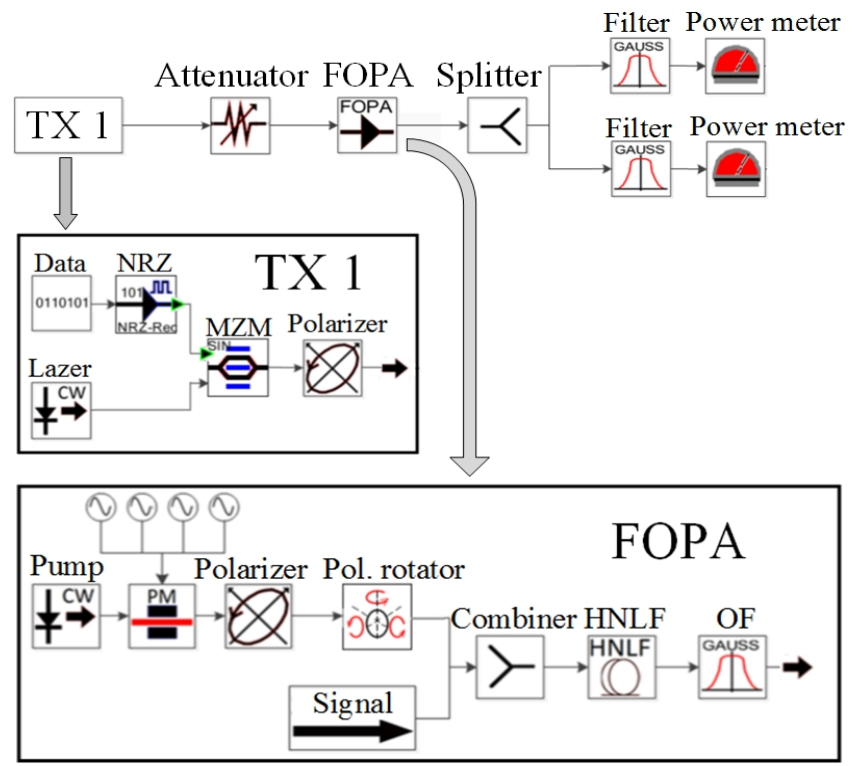

Fig. 1. Simulation model of the single-channel transmission system with a FOPA preamplifier.

In the transmitter the radiation of the continuous wave (CW) optical laser ( $1 \mathrm{~mW} 196.4 \mathrm{THz})$ is externally modulated via a Mach-Zehnder Modulator (MZM), which in its turn is driven by a sequence of NRZ coded electrical pulses that are produced by a tandem of a logical data source and an NRZ coder. At the output of the MZM optical radiation is sent through an optical polarizer in order to ensure that the radiation of the produced signal is linearly polarized and has a certain state of polarization.

At the output of the polarizer the signal is sent through an optical attenuator with $25 \mathrm{~dB}$ attenuation that represents a $125 \mathrm{~km}$ long standard single-mode fiber (SMF) with attenuation of $0.2 \mathrm{~dB} / \mathrm{km}$, and afterwards is processed by a single-pump FOPA.

The pumping radiation of the FOPA $(500 \mathrm{~mW}$ $192.93 \mathrm{THz}$ ) is phase modulated using four radio frequency tunes in order to broaden its spectrum and, therefore, supress SBS. Afterwards, the pump is sent via an optical polarizer, in order to ensure that its radiation is linearly polarised, and then processed by a polarization rotator that was used to control the mutual SOP mismatch between the signal and the pump. After processing through the polarization rotator the pump is combined with the signal and then sent via a $1 \mathrm{~km}$ long high non-linearity fiber (HNLF), the parameters of which are shown in Table I. This HNLF represents the gain medium where the processes of parametric amplification and idler generation takes place.

TABLE I. HNLF PARAMETERS [11]

\begin{tabular}{|c|c|}
\hline Attenuation at $1550 \mathrm{~nm}, \mathrm{~dB} / \mathrm{km}$ & 0.96 \\
\hline Zero dispersion wavelength, $\mathrm{nm}$ & 1553.35 \\
\hline Fiber non-linearity coefficient, $(\mathrm{W} \cdot \mathrm{km})^{-1}$ & 15 \\
\hline Core effective area, $\mu \mathrm{m}^{2}$ & 10 \\
\hline
\end{tabular}

At the output of the FOPA the combination of the amplified signal, the pump, and the generated idler spectral component is divided among two optical power meters by an optical splitter. At the input of these power meter optical filters are placed in order to filter out all of the unnecessary radiation, letting through only the signal or the idler spectral components.

In order to assess the dependencies of the FOPA produced gain and of the generated idler power on the mutual SOP mismatch between the signal and the pump, the pumps radiation was filtered out and optical power of the signal and the idler was obtained at the output of the FOPA for different SOP mismatch values. The obtained results are shown in Fig. 2.

From Fig. 2 it can be seen that the powers of the signal and the idler reached their minimums $(-30.4 \mathrm{dBm}$ and $49.1 \mathrm{dBm}$ respectively) when the SOP of the pump was placed orthogonally in respect to the SOP of the signal, and reached their maximums $(-13.7 \mathrm{dBm}$ and $-15.6 \mathrm{dBm}$ respectively) when the signal and the pump are co-polarised. Taking into account that signal power at the input of the FOPA was $-31 . \mathrm{dBm}$ and that the attenuation of the $1 \mathrm{~km}$ long HNLF fiber was $0.96 \mathrm{~dB}$, it was found that when the signal and the pump were co-polarized FOPA produced gain reached $18.3 \mathrm{~dB}$, but when the $\mathrm{SOP}$ of the signal and of the pump were placed orthogonally - only $1.6 \mathrm{~dB}$. Therefore, the amount of polarization dependant gain (PDG) in this experiment has reached $16.7 \mathrm{~dB}$, but the difference of idler generation efficiency was $33.5 \mathrm{~dB}$.

When both the signal and the pump are linearly polarised and their SOP are placed orthogonally in respect to each other, the signal should not be amplified and the idler should 
not be generated [13]. But the obtained results show that $1.6 \mathrm{~dB}$ amplification was observed and also the idler spectral component was produced. This can be explained by the influence of self-phase modulation (SPM) nonlinearity that occurred during the process of amplification, and by the presence polarization mode dispersion (PMD) in the HNLF. Both SPM and PMD have caused additional phase mismatch between the interacting optical fields and, therefore, have resulted in additional mutual SOP mismatch, thus the SOP of the signal and the SOP of the pump were not exactly orthogonal.

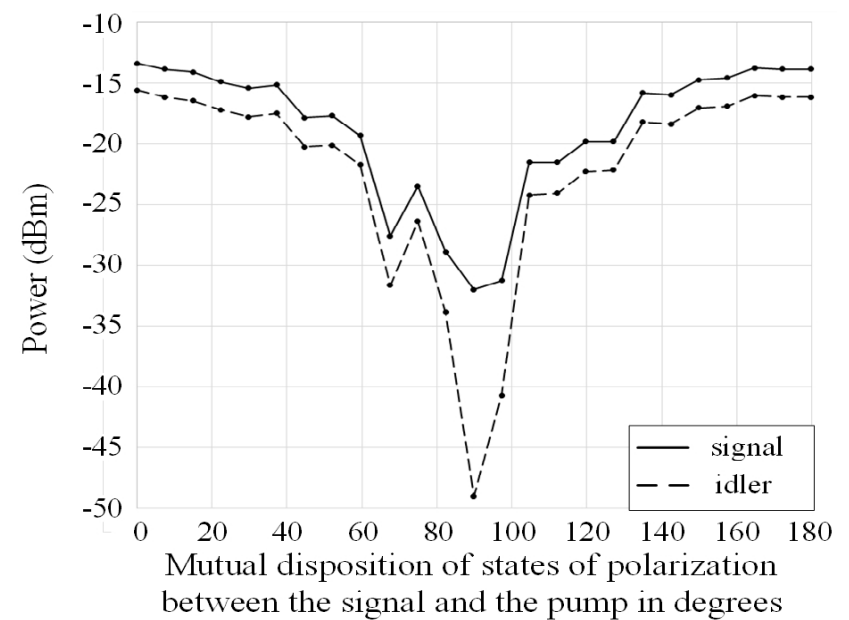

Fig. 2. Dependence of the power of the amplified signal (solid) and of the idler (dashed) at the output of the FOPA on the mutual SOP mismatch between the signal and the pump.

The slight scattering of the results shown in Fig. 2 is explained by the presence of PMD and by the way PMD is realised in the simulation. In real life the value of accumulated PMD varies in time depending on the influence of external factors on the fiber. Therefore in the simulation model for every run (for each point shown in Fig. 2) a new pseudorandom value of PMD is used. Based on the results shown in Fig. 2, it can be concluded, that PMD changes can cause up to $4 \mathrm{~dB}$ gain variations. This is why for implementing FOPAs polarization maintaining HNLF fibers should be used, where the value of PMD does not change under the influence of external factors.

The obtained results indicate that it is vitally important to ensure that the signal and the pump are co-polarised in order to minimise PDG and, therefore, to maximise amplification and idler generation efficiency.

\section{B. Investigation of FOPA Pump Phase Modulation Impact on the Idler Spectral Components}

The most effective way to suppress SBS is to modulate the phase of the pumping radiation, in such a wa broadening its spectrum [14]. As the idler spectral component is a result of FWM interactions between the signal and the pump, and as these parametric interactions are described by the phasematching condition, any phase changes of these two spectral components will directly impact the idler. Therefore, due to the phase sensitivity of FWM, while the spectral width of the signal remains unchanged, any spectral broadening of the pump will cause spectral broadening of the idler in respect to the signal.

To demonstrate the pump phase modulation induced spectral broadening of the idler component and to assess its impact on the quality of the transmitted signal at idler frequencies the same simulation model was used as in the previous research of the authors [11] that was mentioned in the $1^{\text {st }}$ chapter. This simulation scheme is shown in Fig. 3.

The simulation scheme in Fig. 3 is a 9.953 Gbps 8 channel dense WDM (DWDM) transmission system with non-return-to-zero (NRZ) encoding technique, intensity onoff keying modulation format, and an in-line FOPA. The structure of the transmitters and of the parametric amplifier is the same as in Fig. 1. The transmitters operate at frequencies from 196.6 THz to $196.95 \mathrm{THz}$ with $50 \mathrm{GHz}$ channel spacing. The optical radiation of all 8 transmitters is combined via an optical combiner and sent via a $100 \mathrm{~km}$ long SMF, at the end of which, after compensating dispersion using a fiber Bragg grating (FBG), the signal is amplified by a FOPA with $660 \mathrm{~mW} 1553.9 \mathrm{~nm}$ pumping radiation. In order to mitigate the unwanted impact of SBS, the pump phase was modulated using the following four radio-frequency tunes: $180 \mathrm{MHz}, 420 \mathrm{MHz}, 1.087 \mathrm{GHz}$, $2.133 \mathrm{GHz}$. Such configuration of the FOPA was previously used in [11] as it could ensure bit-error ratio (BER) below $10^{-12}$ for all channels at the initial signal frequencies.

The parameters of the optical filter (189.075 center frequency, $650 \mathrm{GHz}-3 \mathrm{~dB}$ two-sided bandwidth) at the output of the amplifier were chosen in a way to filter out the signal and the pumping radiation, thus letting through only the idler spectral components. Therefore, the idlers were used as the informative signal in this experiment. Afterwards the idlers were sent through a $41 \mathrm{~km}$ long SMF, at the end of which, after dispersion compensation, they were divided among 8 receivers.

To estimate the idler spectral broadening, caused by pump-phase modulation, optical spectra of the amplified signal and of the generated idler components were obtained at the output of the FOPA. These results are shown in Fig. 4.

As can be seen in Fig. 4 the spectrum of the idlers is sufficiently wider than of the signal at initial frequencies. To numerally evaluate spectral broadening of the idlers in respect to the initial signal the width of the 5th channel spectrum at $-15 \mathrm{~dB}$ level was obtained for the initial signal and for the idler spectral component.

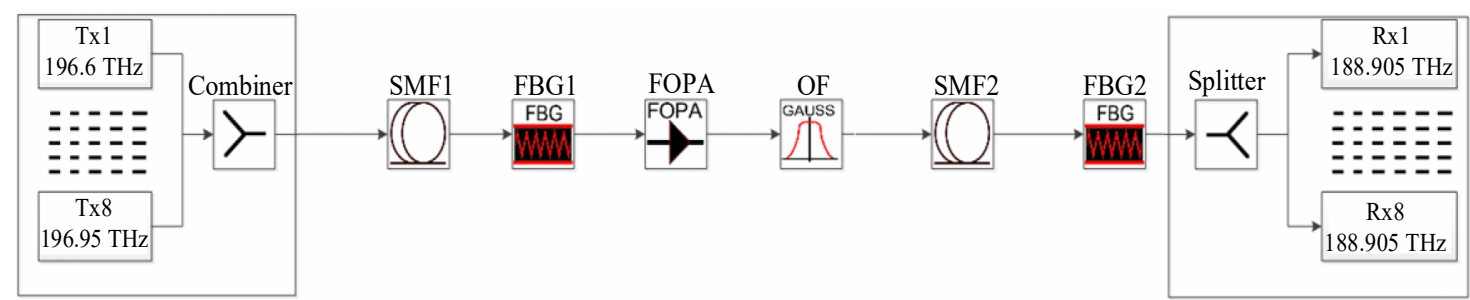

Fig. 3. Simulation model of the 8 channel 9.953 Gbps DWDM transmission system with a FOPA in-line amplifier. 

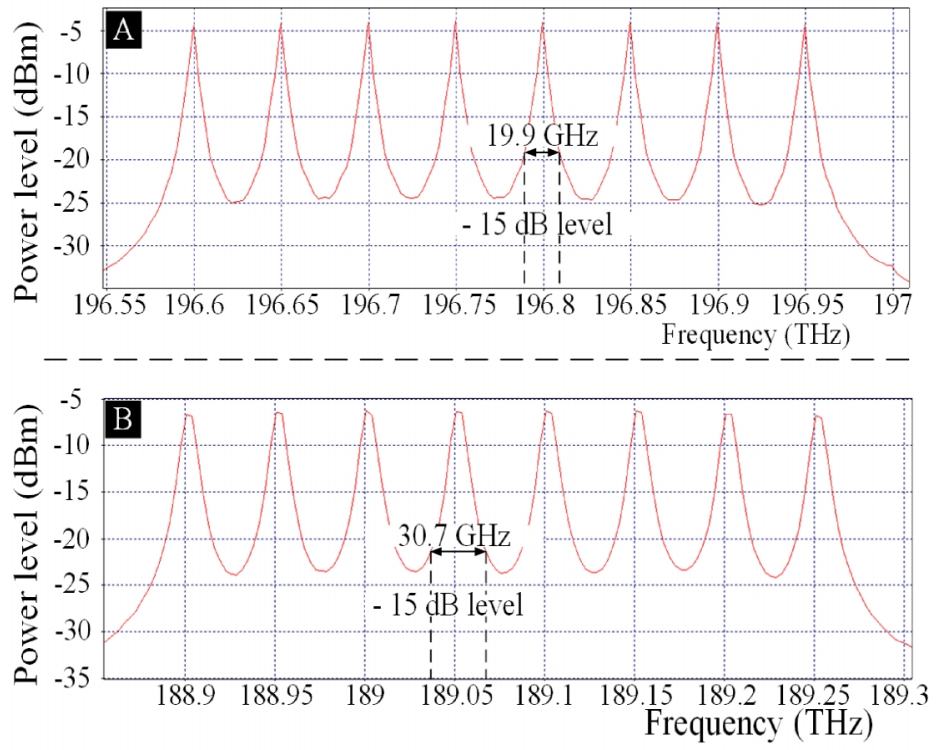

Fig. 4. Spectra of the amplified signal (A) and of the generated idlers (B) at the output of the single-pump FOPA.

The $-15 \mathrm{~dB}$ level was used because, due to spectral broadening, it was not possible to obtain the $-20 \mathrm{~dB}$ level for the idler spectral components. It was found that the idler was by $10.8 \mathrm{GHz}$ wider than the corresponding signal at its initial frequency, therefore $54 \%$ spectral broadening was observed.

In systems where FOPAs are used for wavelength conversion and all-optical signal regeneration such spectral broadening of the idler components is not acceptable as it will cause severe power penalty at the receiver end. After analyzing simulation results it was found that BER value of the signal detected in the $5^{\text {th }}$ channel at idler frequency was at least by 3 orders higher than at signal initial frequency $\left(7.41 \cdot 10^{-10}\right.$ and $5.79 \cdot 10^{-13}$ accordingly). Therefore, while choosing the frequencies of the tones for pump phase modulation it is required to find a compromise between the efficiency of SBS mitigation and idler spectral broadening, or to implement one of the techniques designed to reduce broadening of the idler spectrum [14], [15]. Implementation of such techniques allows to reduce, or even to avoid idler spectral broadening, but it requires high precision of the selected parameters, otherwise the efficiency of FWM will be reduced.

\section{CONCLUSIONS}

The main goal of this article was to estimate the influence of FOPA pump phase modulation and of the mutual SOP mismatch between the signal and the pump at FOPA input on the efficiency and on the spectrum of the generated idler spectral components. To achieve these goals two different simulation models were used.

The first one was a simplified model of a single-channel transmission system that was used to assess the dependence of FOPA idler generation efficiency on the mutual SOP mismatch between the pump and the input signal. $33.5 \mathrm{~dB}$ difference of idler generation efficiency was observed between the cases when the signal and the pump were linearly co-polarized and linearly orthogonally polarized. Surprisingly, it was found that even in the case when the signal and the pump were orthogonally polarized the signal obtained $1.6 \mathrm{~dB}$ gain and idler with $-49.1 \mathrm{dBm}$ power was produced. This is explained by the presence of SPM and PMD that caused additional SOP mismatch between the signal and the pump, therefore, the interacting optical fields were not exactly orthogonally polarised. This additional SOP mismatch can cause high power penalty at the receiver end and, therefore, must be taken into account in systems, where FOPAs are used for demultiplexing of polarization multiplexed signals or for polarization manipulation to intensity OOK modulation format conversion.

The second simulation model was an 8 channel DWDM transmission system with an in-line FOPA, used to estimate how pump phase modulation influences the spectrum of the produced idlers. It was found that such SBS mitigation technique causes the spectra on the idlers to broaden - $54 \%$ spectral broadening was observed in the $5^{\text {th }}$ channel. Such idler spectral broadening will cause severe degradation of signal quality at the receiver in systems where FOPAs are used for wavelength conversion, all-optical regeneration, or TDM signal demultiplexation, as it produces severe interchannel crosstalk in the neighbour channels. Therefore, in such systems special measures must be taken to avoid idler spectral broadening.

\section{REFERENCES}

[1] P. Sharma, A. Kumar, V. K. Sharma, "Performance analysis of high speed optical network based on Dense Wavelength Division Multiplexing", Int. Conf. Issues and Challenges in Intelligent Computing Techniques (ICICT), 2014, pp. 446-449. [Online]. Available: http://dx.doi.org/10.1109/icicict.2014.6781323

[2] S. Olonkins, S. Spolitis, I. Lyashuk, V. Bobrovs, "Cost effective WDM-AON with multicarrier source based on dual-pump FOPA", 6th Int. Congress on Ultra Modern Telecommunications and Control Systems and Workshops (ICUMT), 2014, pp. 23-28. [Online]. Available: http://dx.doi.org/10.1109/icumt.2014.7002073

[3] N. Sharma, R. Vij, N. Badhan, "Enhanced spectral efficiency for intensity modulated DWDM systems", Twenty First National Conf. Communications (NCC 2015), 2015, pp. 1-6. [Online]. Available: http://dx.doi.org/10.1109/ncc.2015.7084818

[4] S. Olonkins, I. Lyashuk, V. Bobrovs, G. Ivanovs, "Equalization of EDFA Gain spectrum and increase of OSNR through introducing a hybrid Raman-EDFA solution", Progress in Electromagnetics 
Research Symposium (PIERS 2015), 2015, pp. 600-603.

[5] M. C. Ho, M. E. Marhic, Y. Akasaka, L. G. Kazovsky, "Fiber optical parametric amplifier with $208 \mathrm{~nm}$ gain bandwidth", Conf. Lasers and Electro-Optics, 2000, pp. 401-402.

[6] T. Torouinidis, P. A. Andrekson, B. E. Olsson, "Fiber-optical parametric amplifier with 70-dB gain", IEEE Photonics Technology Letters, vol. 18, no. 10, pp. 1194-1196, 2006. [Online]. Available: http://dx.doi.org/10.1109/LPT.2006.874714

[7] T. Sylvestre, A. Mussot, A. Vedadi, L. Provivo, E. Lantz, H. Maillotte, "System performance of fiber optical amplifiers", Fiber and Integrated Optics, vol. 27, no. 6, pp. 516-531, 2008. [Online]. Available: http://dx.doi.org/10.1080/01468030802269787

[8] J. Wang, J. Yu, T. Meng, W. Miao, et al. "Simultaneous 3R regeneration of $4 * 40-\mathrm{Gbit} / \mathrm{s}$ WDM signals in a single fiber", IEEE Photonics Journal, vol. 4, no. 5, pp. 1816-1822, 2012. [Online]. Available: http://dx.doi.org/10.1109/JPHOT.2012.2215955

[9] M. Karasek, P. Honzatko, J. Vojtech, J. Radil, "Multi-wavelength conversion at $10 \mathrm{~Gb} / \mathrm{s}$ and $40 \mathrm{~Gb} / \mathrm{s}$ based on 2 pumps FOPA", 13th Int. Conf. Transparent Optical Networks (ICTON 2011), 2011, pp. 1-4. [Online]. Available: http://dx.doi.org/10.1109/ICTON.2011. 5970951

[10] L. Ruo-Ding, P. Kumar, W. L. W. Kath, "Dispersion compensation with phase-sensitive optical amplifiers", IEEE Journal of Lightwave Technology, no. 12, pp. 541-549, 1994. [Online]. Available: http://dx.doi.org/10.1109/50.285338

[11] S. Olonkins, V. Bobrovs, G. Ivanovs, "Investigation of fiber optical parametric amplifier performance in DWDM transmission systems", Elektronika Ir Elektrotechnika, vol. 20, no. 1, pp. 88-91, 2014. [Online]. Available: http://dx.doi.org/10.5755/j01.eee.20.1.6170

[12] OptSim 5.3 User Guide, RSoft Design Group. [Online]. Available: http://www.rsoftdesign.com

[13] Q. Lin, G. P. Agrawal, "Vector theory of four-wave mixing. polarization effects in fiber-optic parametric amplifiers", Journal of the Optical Society of America, vol. 21, pp. 1216-1224, 2004. [Online]. Available: http://dx.doi.org/10.1364/JOSAB.21.001216

[14] T. Tanemura, C. H. Lim, K. Kikuchi, "Suppression of idler spectral broadening in highly efficient fiber four-wave mixing by binaryphase-shift-keying modulation of pump wave", in Photonics Technology Letters, IEEE, vol. 13, no. 12, pp. 1328-1330, 2001. [Online]. Available: http://dx.doi.org/10.1109/68.969897

[15] S. Yamashita, K. Torii, "Cancellation of spectral spread in highly efficient optical fiber wavelength converters", Electron. Lett., vol. 36, pp. 1997-1998, 2000. [Online]. Available: http://dx.doi.org/10.1049/ el:20001387 